\title{
Finite-size effects and the search for the critical endpoint of QCD
}

\author{
Eduardo Souza Fraga* \\ Instituto de Física, Universidade Federal do Rio de Janeiro, \\ Caixa Postal 68528, Rio de Janeiro, RJ 21941-972, Brazil \\ E-mail: fragaeif.ufrj.br
}

\section{Takeshi Kodama}

Instituto de Física, Universidade Federal do Rio de Janeiro, Caixa Postal 68528, Rio de Janeiro, RJ 21941-972, Brazil

E-mail: tkodama@if.ufrj.br

\section{Letícia F. Palhares}

Instituto de Física, Universidade Federal do Rio de Janeiro, Caixa Postal 68528, Rio de Janeiro, RJ 21941-972, Brazil

Institut de Physique Théorique, CEA/DSM/Saclay,

Orme des Merisiers, 91191 Gif-sur-Yvette cedex, France

E-mail: leticialif.ufrj.br

\section{Paul Sorensen}

Physics Department, Brookhaven National Laboratory,

Upton, NY 11973-5000, USA

E-mail: psoren@bnl.gov

\begin{abstract}
Taking into account the finiteness of the system created in heavy ion collisions, we show sizable results for the modifications of the chiral phase diagram at volume scales typically encountered in current experiments and demonstrate the applicability of finite-size scaling as a tool in the experimental search for the critical endpoint. Using data from RHIC and SPS and assuming finite-size scaling, we find that RHIC data from $200 \mathrm{GeV}$ down to $19.6 \mathrm{GeV}$ is only consistent with a critical point at $\mu \gtrsim 510 \mathrm{MeV}$. We also present predictions for the fluctuations at lower energies currently being investigated in the Beam Energy Scan program.
\end{abstract}

The many faces of $Q C D$

November 1-5, 2010

Gent, Belgium

\footnotetext{
${ }^{*}$ Speaker.
} 
Relativistic heavy ion collision experiments have entered a new era of investigations of the phase diagram of strong interactions. The Beam Energy Scan program at RHIC [1] is probing a relatively wide window in center-of-mass energy, which translates into higher values of the baryonic chemical potentials and lower values of the temperature associated with the plasma created in the collision process. The hope is that the scan will able to reach the region where a second-order critical point is expected to exist [2]. Therefore, pragmatic theoretical tools that can be phenomenologically applied to data analysis are demanded.

Identifying the presence of a critical point, and even of a first-order transition coexistence line, is not a simple task in heavy ion experiments. One has to uncover, from different sorts of complex backgrounds, signatures related to large fluctuations that would stem from the critical behavior of the order parameter of the chiral transition, the chiral condensate. In a system in equilibrium and in the thermodynamic limit, those are a consequence of the unlimited growth of the correlation length [3], and in the case of heavy ions would lead to non-monotonic behavior [4, 5] or sign modifications [6] of particle correlation fluctuations.

Although often compared to the case of the early universe at the time of the primordial quarkhadron transition, the space-time scales present in the dynamics of the quark-gluon plasma formed in heavy ion collisions differ from the cosmological ones by almost twenty orders of magnitude. So, realistically, this system is usually small, short-lived, and part of the time out of equilibrium. The finite (short) lifetime of the plasma state and critical slowing down could severely constrain the growth of the correlation length, as shown by estimates in Refs. [7, 8]. The finite size of the system could dramatically modify the phase structure of strong interaction, as shown using lattice simulations $[9,10]$ and different effective model approaches [11, 12, 13, 14], and also affect significantly the dynamics of phase conversion $[15,16]$. As a consequence, all signatures of criticality based on non-monotonic behavior of particle correlation fluctuations will probe a pseudocritical point that can be significantly shifted from the genuine (unique) critical endpoint by finite-size corrections and will be sensitive to boundary effects.

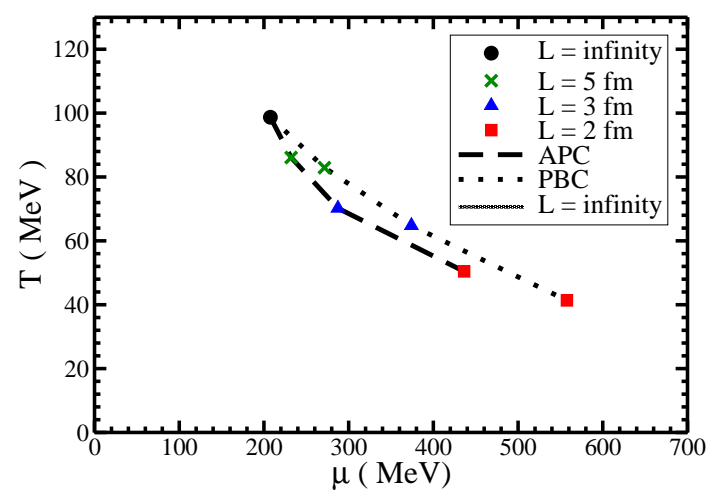

Figure 1: Displacement of the pseudocritical endpoint in the $T-\mu$ plane as the system size is decreased for different boundary conditions.

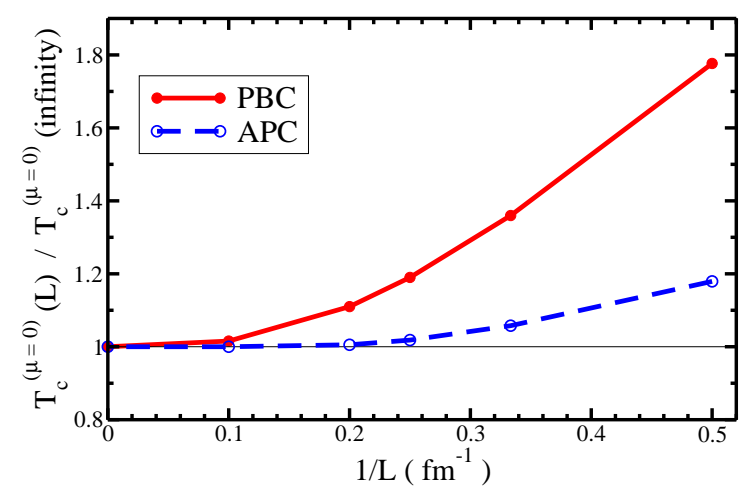

Figure 2: Normalized crossover temperature at $\mu=0$ as a function of the inverse size $1 / L$ for the cases with PBC and APC.

The latter effect was demonstrated using the linear sigma model coupled to quarks with two flavors [17] as an effective theory for the chiral transition in Ref. [14]. Here we illustrate the rele- 
vance the effects coming from the finite size of the system, of typical linear size $L$, and the nature of the boundary might have in the investigation of the phase diagram of strong interactions using heavy ion collisions in Figs. 1 and 2. Fig. 1 shows the displacement of the pseudocritical point, comparing periodic boundary conditions (PBC) and anti-periodic boundary conditions (APC): both coordinates of the critical point are significantly modified, and $\mu_{\mathrm{CEP}}$ is about $30 \%$ larger for PBC. For $\mu=0$, the crossover transition is also affected by finite-size corrections, increasing as the system decreases, as shown in Fig. 2. Again, PBC generate larger effects: up to $\sim 80 \%$ increase in the crossover transition temperature at $\mu=0$ when $L=2 \mathrm{fm}$. The range of values for the linear size $L$ is motivated by the estimated plasma size presumably formed in high-energy heavy ion collisions at RHIC [18]. The upper limit is essentially geometrical, provided by the radius of the nuclei involved, whereas the lower limit is an estimate for the smallest plasma observed.

Nevertheless, the finiteness of the system in heavy ion collisions also brings a bright side: the possibility of using finite-size scaling (FSS) analysis [19, 20, 21]. FSS is a powerful statistical mechanics technique that prescinds from the knowledge of the details of a given system; instead, it provides information about its criticality based solely on very general characteristics. And since the thermal environment corresponding to the region of quark-gluon plasma formed in heavy ion collisions can be classified according to the centrality of the collision, events can be separated according to the size of the plasma that is created. So, heavy ion collisions indeed provide an ensemble of differently-sized systems.

Although it is clearly not simple to define an appropriate scaling variable in the case of heavy ion collisions, the flexibility of the FSS method allows for a pragmatic approach for the use of scaling plots in the search for the critical endpoint as was delineated in Ref. [14] and performed in Ref. [22]. The essential point is that although the reduced volume of the plasma formed in high-energy heavy ion collisions will dissolve a possible critical point into a region and make the effects from criticality severely smoothened, as discussed above, the non-monotonic behavior of correlation functions for systems of different sizes, given by different centralities, must obey FSS near criticality $[3,23]$.

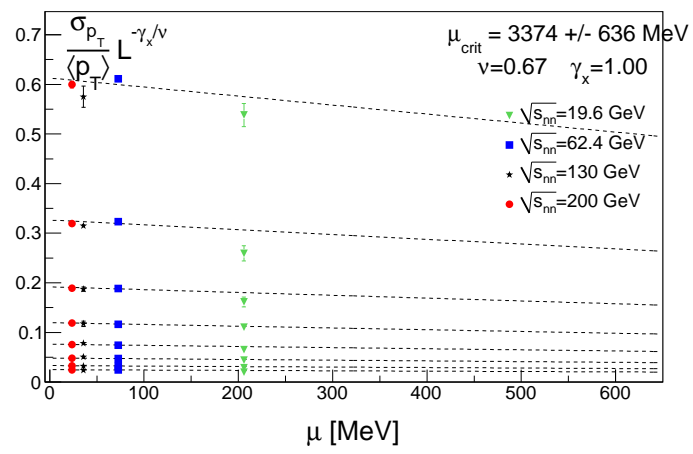

Figure 3: Scaled $\sigma_{p_{T}} /\left\langle p_{T}\right\rangle$ vs $\mu$ for different system sizes, and with $v=2 / 3$ and $\gamma_{x}=1$. Data extracted from RHIC collisions at energies $\sqrt{s_{N N}}=$ 19.6, 62.4, 130, and $200 \mathrm{GeV}$ (linear fit, see text).

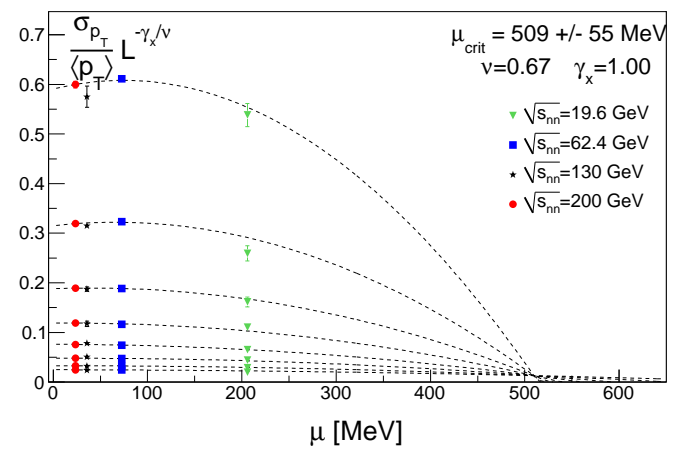

Figure 4: Scaled $\sigma_{p_{T}} /\left\langle p_{T}\right\rangle$ vs $\mu$ for different system sizes. Again, $v=2 / 3$ and $\gamma_{x}=1$. Data extracted from RHIC collisions at energies $\sqrt{s_{N N}}=19.6,62.4,130$, and $200 \mathrm{GeV}$ (second order polynomial fit, see text). 
Using data from RHIC and SPS and defining appropriate scaling variables, we can generate scaling plots for data sets with $\sqrt{s_{N N}}=17.3,19.6,62.4,130,200 \mathrm{GeV}$. For finite $L$, crossover effects become important. If the correlation length diverges as $\xi_{\infty} \sim t^{-v}$ at criticality, where $v$ is the corresponding critical exponent, in the case of $L^{-1} t^{-v} \gg 1$ the system is no longer governed by the critical fixed point and $L$ limits the growth of the correlation length, rounding all singularities [3,23]. If $L$ is finite, $\xi$ is analytic in the limit $t \rightarrow 0$, and one can draw scaling plots of $L / \xi$ vs. some coupling for different values of $L$ to find that all curves cross at a given value in this limit, which is a way to determine its critical value. The critical temperature and so on can also be determined in this fashion, since the curves will also cross at $t=t_{c}$. This scaling plot technique can be extended, taken to its full power for other quantities, such as correlation functions. An observable $X$ in a finite thermal system can be written, in the neighborhood of criticality, in the following form [21]:

$$
X(t, L)=L^{\gamma_{x} / v} f\left(t L^{1 / v}\right),
$$

where $\gamma_{x}$ is the bulk (dimension) exponent of $X$ and $\{g\}$ dimensionless coupling constants. The function $f(y)$ is universal up to scale fixing, and the critical exponents are sensitive essentially to dimensionality and internal symmetry, which will give rise to the different universality classes [23]. Using the appropriate scaling variable, all curves should collapse into one single curve if one is not far from the critical point. So, this technique can be applied to the analysis of observables that are directly related to the correlation function of the order parameter of the transition, such as fluctuations of the multiplicity of soft pions [4]. The correct scaling variable should measure the distance from the critical point, thereby involving both temperature and chemical potential in the case of the QCD phase diagram. This would produce a two-dimensional scaling function and make the analysis of heavy ion data highly nontrivial. Phenomenologically, we adopt a simplification motivated by results from thermal models for the freeze-out region, connecting temperature and chemical potential. We can parametrize the freeze-out curve by $\sqrt{s_{N N}}$, and build our one-dimensional scaling variable from this quantity and the size of the system. For details, we refer the reader to Ref. [22].

To search for scaling, we consider the correlation measure $\sigma_{p_{T}} /\left\langle p_{T}\right\rangle$ [24] scaled by $L^{-\gamma_{x} / v}$, according to Eq. 1 . We consider the $p_{T}$ fluctuations $\sigma_{p_{T}}$ scaled by $\left\langle p_{T}\right\rangle$ to obtain a dimensionless variable. We use the correlation data measured in bins corresponding to the $0-5 \%, 5-10 \%$, $10-20 \%, 20-30 \%, 30-40 \%, 40-50 \%, 50-60 \%$, and $60-70 \%$ most central collisions. We estimate the corresponding lengths $L$ to be $12.4,11.1,9.6,8.0,6.8,5.6,4.5$, and $3.4 \mathrm{fm}$. The exponent $v=2 / 3$ is determined by the Ising universality class of QCD and we consider values of $\gamma_{x}$ around 1 (ignoring small anomalous dimension corrections). We also varied the value of $\gamma_{x}$ from 0.5 to 2.0 and found that changing $\gamma_{x}$ within this range does not improve the scaling behavior.

In Figs. 3 and 4 we plot $\sigma_{p_{T}} /\left\langle p_{T}\right\rangle$ scaled by $L^{-\gamma_{x} / v}$ vs $\mu$ for different system sizes, using data extracted from collisions at $\sqrt{s_{N N}}=19.6,62.4,130,200 \mathrm{GeV}$. If there is a critical point at $\mu=\mu_{\text {crit }}$, the curves for different sizes of the system should cross at this value of $\mu$. However, since the currently available data is restricted to not so large values of the chemical potential, one has to perform extrapolations using fits. The scaling function $f$ in Eq. (1) is expected to be smoothly varying around the critical point, so we fit the data corresponding to a given linear size $L$ to a polynomial, but constraining the polynomials to enforce the condition that all the curves cross at some $\mu=\mu_{\text {crit }}$, where $\mu_{\text {crit }}$ is an adjustable parameter in the fit. This clearly assumes the existence of a critical point. In Fig. 3 we use a linear fit. The approximate energy independence of $\sigma_{p_{T}} /\left\langle p_{T}\right\rangle$ 
along with the linear fit, leads to a very large $\mu$ value where the curves can cross $(\mu \sim 3 \mathrm{GeV})$. There is no reason however to assume a linear fit function, so in Fig. 4 we also try a second order polynomial. The assumption of a second order polynomial function for $f$ allows the curves from different system sizes to cross at a much smaller value of $\mu$. Based on this fit, we find that the data is consistent with a critical point at $\mu \sim 510 \mathrm{MeV}$ corresponding to a $\sqrt{s_{N N}}$ of $5.75 \mathrm{GeV}$.

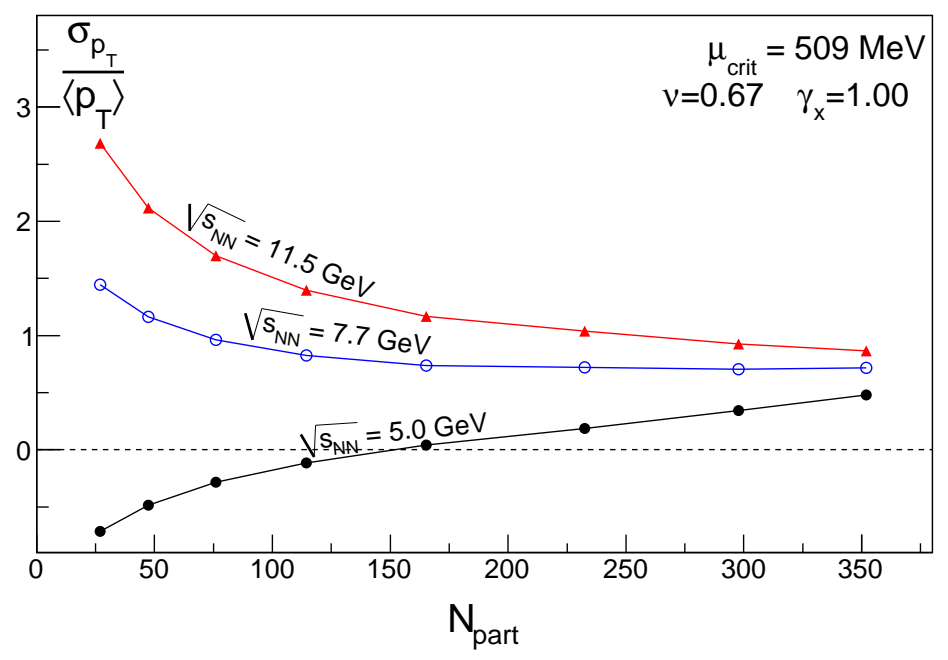

Figure 5: The expected measurement of $\sigma_{p_{T}} /\left\langle p_{T}\right\rangle$ as a function of the number of participants at lower energies assuming the critical point is at $509 \mathrm{MeV}$ as extracted from the quadratic polynomial fit of STAR data.

RHIC has also run at lower energies in order to search for a critical point in the Beam Energy Scan program. Using the quadratic polynomial fit of STAR data (Fig. 4) and assuming the critical point is at $509 \mathrm{MeV}$ we can make predictions for $\sigma_{p_{T}} /\left\langle p_{T}\right\rangle$ at lower energies. We show this expectation as a function of the number of participants, $N_{\text {part }}$, for three proposed beam energies: 11.5,7.7 and $5 \mathrm{GeV}$ in Fig. 5. Notice that the centrality dependence changes once one moves to the other side of the critical point. This is a condition enforced by finite-size scaling which provides a generic signal for having reached the first-order phase transition side of the critical point.

Finite size effects are non negligible in heavy ion experiments and the mapping of the phase diagram for QCD they can produce. In fact, most thermodynamic quantities will be considerably shifted in the pseudocritical diagram that is actually probed, so that comparisons to results from the lattice in the thermodynamic limit should be made with caution. On the other hand, finitesize scaling techniques are simple and well defined in the case of heavy ion collisions. Even if it is hard to define the ideal scaling variable, it provides a pragmatic method to search for the critical point and investigate the universality properties of QCD. The fact that FSS prescinds from the knowledge of the details of the system under consideration, providing information about its criticality based solely on its most general features, makes it a very powerful tool for data analysis. From a very limited data set in energy spam, we have used FSS to exclude the presence of a critical point at small values of the baryonic chemical potential, below $450 \mathrm{MeV}$. Besides, FSS is naturally fit to produce concrete predictions: once one assumes the existence of criticality, the scaling of correlation functions must be manifest in its vicinity. 
We are grateful to M. Chernodub, S.L.A. de Queiroz and Á. Mócsy for fruitful discussions. This work was partially supported by CAPES-COFECUB (project 663/10), CNPq, FAPERJ and FUJB/UFRJ.

\section{References}

[1] B. I. Abelev et al. [STAR Collaboration], STAR Note 0493 (2009); M. M. Aggarwal et al. [STAR Collaboration], arXiv:1007.2613 [nucl-ex].

[2] J. Berges and K. Rajagopal, Nucl. Phys. B 538, 215 (1999); A. M. Halasz, A. D. Jackson, R. E. Shrock, M. A. Stephanov and J. J. M. Verbaarschot, Phys. Rev. D 58, 096007 (1998).

[3] J. L. Cardy, Scaling and Renormalization in Statistical Physics (Cambridge, 1996).

[4] M. A. Stephanov, K. Rajagopal and E. V. Shuryak, Phys. Rev. Lett. 81, 4816 (1998); Phys. Rev. D 60, 114028 (1999).

[5] M. A. Stephanov, Phys. Rev. Lett. 102, 032301 (2009).

[6] M. Asakawa, S. Ejiri and M. Kitazawa, Phys. Rev. Lett. 103, 262301 (2009).

[7] B. Berdnikov and K. Rajagopal, Phys. Rev. D 61, 105017 (2000).

[8] M. A. Stephanov, Phys. Rev. D 81, 054012 (2010).

[9] A. Gopie and M. C. Ogilvie, Phys. Rev. D 59, 034009 (1999).

[10] A. Bazavov and B. A. Berg, Phys. Rev. D 76, 014502 (2007).

[11] O. Kiriyama and A. Hosaka, Phys. Rev. D 67, 085010 (2003); O. Kiriyama, T. Kodama and T. Koide, arXiv:hep-ph/0602086; L. M. Abreu, M. Gomes and A. J. da Silva, Phys. Lett. B 642, 551 (2006); L. M. Abreu, A. P. C. Malbouisson, J. M. C. Malbouisson, A. E. Santana, Nucl. Phys. B819, 127-138 (2009); L. M. Abreu, A. P. C. Malbouisson, J. M. C. Malbouisson, Phys. Rev. D83, 025001 (2011).

[12] J. Braun, B. Klein and H. J. Pirner, Phys. Rev. D 71, 014032 (2005); Phys. Rev. D 72, 034017 (2005); J. Braun, B. Klein, H. J. Pirner and A. H. Rezaeian, Phys. Rev. D 73, 074010 (2006).

[13] N. Yamamoto and T. Kanazawa, Phys. Rev. Lett. 103, 032001 (2009).

[14] L. F. Palhares, E. S. Fraga and T. Kodama, arXiv:0904.4830 [nucl-th] (J. Phys. G, in press); PoS CPOD2009, 011 (2009); J. Phys. G G37, 094031 (2010).

[15] C. Spieles, H. Stoecker and C. Greiner, Phys. Rev. C 57, 908 (1998).

[16] E. S. Fraga and R. Venugopalan, Physica A 345, 121 (2004).

[17] M. Gell-Mann and M. Levy, Nuovo Cim. 16, 705 (1960).

[18] J. Adams et al. [STAR Collaboration], Nucl. Phys. A 757, 102 (2005).

[19] M. E. Fisher, in Critical Phenomena, Proc. 51st Enrico Fermi Summer School, Varena, ed. M. S. Green (Academic Press, NY, 1972); M. E. Fisher and M. N. Barber, Phys. Rev. Lett. 28, 1516 (1972).

[20] E. Brezin, J. Physique 43, 15 (1982).

[21] E. Brezin and J. Zinn-Justin, Nucl. Phys. B 257, 867 (1985).

[22] E. S. Fraga, L. F. Palhares and P. Sorensen, arXiv:1104.3755 [hep-ph] (Phys. Rev. C, in press).

[23] D. Amit, Field Theory; The Renormalization Group and Critical Phenomena (World Scientific, 2005).

[24] J. Adams et al. [STAR Collaboration], Phys. Rev. C 72, 044902 (2005). 